\title{
Évaluation de l'âge optimal de maturation des différentes variétés de manioc (Manihot esculenta Crantz) tant locales qu'améliorées cultivées à Ngandajika en République Démocratique du Congo
}

\author{
${ }^{1}$ Mukendi Tshizembe Donat, ${ }^{1}$ Tshimbombo Jadika Carcy, ${ }^{2}$ Muyayabantu Mupala George, ${ }^{1}$ Tshiamala \\ Ngeleka Théophile., 'Kamukenji Nam'a Mbaji Alphonse, 'Beya Mutombo Salomon et ${ }^{1}$ Mukendi \\ Kamambo Robert \\ ${ }^{1}$ Institut National pour Étude et Recherche Agronomique, Centre de Recherche de Ngandajika, \\ 2 Université Officielle de Mbuji-Mayi. (U.O.M.) \\ Email correspondant : mukendidonat@yahoo.fr et/ou donnamukendi@gmail.com
}

Original submitted in on 24th October 2017. Published online at www.m.elewa.org on $31^{\text {st }}$ January 2018 https://dx.doi.org/10.4314/jab.v121i1.3

\section{RÉSUMÉ}

Objectif: Une étude a été menée à la station de l'Institut National pour l'Étude et la Recherche Agronomique (INERA) de Ngandajika, province de la Lomami, République Démocratique du Congo, durant les années culturales 2013/2014 et 2014/2015 en vue de déterminer l'âge optimal de récolte des différentes variétés de manioc locales et améliorées cultivées dans cette partie du pays.

Méthodologie et résultats: Treize variétés de manioc dont 8 améliorées (Nsansi, Butamu, Mbankana, Zizila, Obama, Vuvu, Ngandajika et Mvuazi) et 5 témoins locales (Kamana Mabanza, Lueni, Kabuitshi, Kalenda et Tshilobo) ont été évaluées dans un essai installé suivant le dispositif des blocs complètement randomisés avec 3 répétitions. 9 plants de manioc étaient récoltés par parcelle à l'intervalle de 3 mois à partir de 9 Mois Après Plantation (9MAP) jusqu'à 21MAP, pour la détermination de rendement en tubercules frais, de la teneur en matière sèche et en amidon. Malgré les différences significatives observées entre les performances de ces variétés, on a noté en général que les moyennes des quatre paramètres évalués ont manifesté une tendance à la croissance jusqu'à 15 MAP. Les moyennes les plus élevées de rendement en tubercules frais (41,3 t/ha), de rendement $\sec (13,7 \mathrm{t} / \mathrm{ha})$, de la Matière sèche $(33,2 \%)$, de l'amidon $(17,8 \%)$ ont été observées à 15 MAP.

Conclusion et application des résultats : Les résultats obtenus ont montré que la période optimale pour la récolte de toutes ces variétés se situerait entre 12 et $15 \mathrm{MAP}$. Nous recommandons que ces variétés locales et améliorées soient récoltées tout au plus tard à 15 MAP.

Mots clés : Manioc, variétés, âge de récolte. 


\section{ABSTRACT}

Objective: A study was conducted at the Centre of the National Institute for the study and the Agronomic Research (INERA) of Ngandajika, province of the Lomami, Democratic Republic of Congo, during the 2years cultural season (2013/2014 and 2014/2015) in order to determine the optimal age of harvest of the local and improved different main varieties of cassava cultivated in this part of the country.

Methodology and Results: Thirteen varieties of cassava of which 8 improved (Nsansi, Butamu, Mbankana, Zizila, Obama, Vuvu, Ngandajika and Mvuazi) and 5 check or local (Kamana Mabanza, Lueni, Kabuitshi, Kalenda and Tshilobo) have been evaluated in a test installed according to the device of the blocks completely randomized with 3 spatial repetitions. The 9 plants of cassava were quarterly harvested by parcel from 9 Months after Plantation (9MAP) until 21MAP, for the determination of cassava fresh tubers yield, the dry matter and the starch. Despite of the meaningful differences observed between the performances of these varieties, one noted in general that the means of the four estimated parameters showed a tendency to grow until 15 MAP. The most elevated means: of output in fresh tubers (41. $3 \mathrm{t} / \mathrm{ha})$, of dry yield output (13, $7 \mathrm{t} / \mathrm{ha})$, of the dry Matter (33.2\%), of the starch (17.8\%) were observed in 15 MAP. Conclusion and application of results: The results obtained showed that the optimum period for the harvest of all these varieties would be between 12 and 15 MAP. We recommend that these local and improved varieties be harvested at the latest by 15 MAP.

Keywords: cassava, varieties, harvest period

\section{INTRODUCTION}

Le manioc (Manihot esculenta Crantz) est cultivé essentiellement pour ses racines tubérisées et, parfois pour ses feuilles. II entre ainsi pour une grande part dans l'alimentation de nombreuses populations dans les zones tropicales d'Amérique, d'Afrique et d'Asie, soit comme source principale d'hydrate de carbone, soit encore comme un apport complémentaire de vitamines, de minéraux et de protéines. La culture de manioc est la deuxième culture vivrière de base en Afrique Subsaharienne où le manioc joue un grand rôle dans la sécurité alimentaire et fournit plus de 285 calories par personnes par jour (Dénis Fargette et al. 1998, Benesi et al. 2004). Quotidiennement, en République Démocratique du Congo, le manioc fournit près de $60 \%$ des calories, à plus de $70 \%$ des populations congolaises (Tata Hangy et Phemba Phezo, 2002). La récolte a lieu entre 7 et 18 mois après plantation, mais les racines peuvent se conserver encore longtemps dans le sol et constituer ainsi une bonne production durant la période de soudure (Dénis Fargette et al, 1988). Selon la durée de conservation en terre de la qualité de leurs racines, les variétés de manioc sont classées en variétés précoce (maturité de 912 mois), semi-tardives (maturité de 15-18 mois) et variétés tardives (maturité de 18-24 mois) (Indira J.
E. et Omar L., 2003 ; IITA, 2012 ; Bassey q. E. et G.I. Harry, 2013). Une récolte précoce conduit généralement à une réduction de rendement en racines tubéreuses tandis que lorsqu'elle est tardive, les racines tubéreuses se lignifient et regorgent beaucoup de fibres. Le taux d'amidon diminue et la qualité des racines se détériore (Tawurunga et al., 1998; IITA DRC, 2012, Tewodros M 2012 E. Bassey et G.I. Harry, 2013 ). Après le développement complet de la canopée foliaire, la croissance des racines tubéreuses diminue progressivement jusqu'à devenir nulle. $\mathrm{Ce}$ moment est appelé la période de maturité du manioc (Okonkwo, 2002). C'est le moment où le maximum ou le quasi maximum de rendement est obtenu. Plusieurs études ont été menées pour déterminer l'âge optimum de maturité de manioc en déterminant le rendement maximum en tubercules frais à travers les expériences (Hahn et al. 1979, Ashoka et al. 1984, Mani et al., 1997, Tewodoros M. et al., 2012, Michael A.Edet et al., 2015). Cependant, leurs recommandations sont variées. En République Démocratique du Congo (RDC), avant 2011, au cours de leur développement, les variétés améliorées de manioc étaient évaluées communément sur une période de 12 mois alors qu'actuellement les variétés sont 
évaluées à 24 mois. Ainsi, toutes les variétés actuellement en diffusion n'ont pas été caractérisées par rapport à la période de leur maturité. Par ailleurs, les producteurs de manioc récoltent leurs champs (variétés locales et améliorées) avant et/ou au-delà de cette période. A Ngandajika, chez les agriculteurs traditionnels, la récolte se fait graduellement et peut même dépasser 21 mois (compers Mukendi). Dans ce territoire, les agriculteurs qui travaillent dans l'hinterland de la cité de Ngandajika, où le problème de terre se pose préfèrent les variétés précoces qu'ils peuvent récolter et/ou vendre endéans 12 mois afin de libérer la terre pour le semis de maïs, tandis que les paysans des villages éloignés et qui ont suffisamment des terres, préfèrent des variétés semi-tardives ou tardives qu'ils peuvent récolter de manière échelonnée selon les besoins de la famille. L'adoption des variétés diffusées varie ainsi dans ces différents milieux selon la préférence du producteur. L'objectif poursuivi dans cette étude,

\section{MATÉRIEL ET MÉTHODE}

Site d'étude: L'étude s'est déroulée au centre de recherche de l'Institut National pour l'étude et la Recherche Agronomique (INERA) de Ngandajika, pendant deux années agricoles : 2013-2014 et 20142015. Le territoire de Ngandajika (Province de Lomami), a les coordonnées géographiques suivantes: $6^{\circ} 45^{\prime} 46^{\prime \prime}$ latitude S, 23 57' 14" longitude E et $790 \mathrm{~m}$ d'altitude (M. Muengula M, 2010). Le climat du milieu est du type $\mathrm{Aw}_{3}$ suivant la classification climatique de Köppen, caractérisé par l'alternance de deux saisons (la saison pluvieuse et la saison sèche). La répartition annuelle de pluie est bimodale avec le pic en janvier et en Avril. La pluviométrie annuelle varie de 1400 à $1500 \mathrm{~mm}$ de pluie, la température annuelle moyenne est d'environ $24^{\circ}$. Selon les informations tirées de l'IRAZ (1989), la texture du sol de Ngandajika est sablo-argileuse à argile lourd, de coloration rouge à ocre rouge, et de bonne structure. La fraction argileuse est peu importante et varie selon les localités. Le pH du sol varie de 5.2 à 6.8. Les données géographiques du site sont: $6^{\circ} 48^{\prime} 33^{\prime \prime}$ latitude sud, $23^{\circ} 57^{\prime} 04^{\prime \prime}$ longitude $E$ et $746 \mathrm{~m}$ d'altitude.

Matériel: Huit variétés de manioc améliorées et résistantes à la Mosaïque Africaine dont Mbankana, Ngandajika, Nsansi, Zizila, Butamu, Mvuazi, Vuvu, est d'évaluer la période de maturité optimale de chacune des variétés de manioc cultivées en vue d'aider le paysan à faire son choix selon sa préférence. L'hypothèse émise est que les variétés diffusées n'atteignent pas toutes la maturité au même moment et qu'il serait possible, en évaluant l'évolution de leur rendement en tubercules frais et de leur teneur en Matière sèche, de déterminer celles qui sont précoces, semitardives ou tardives. Dans cet essai, les principales variétés améliorées et locales de manioc cultivées à travers le territoire de Ngandajika ont été évaluées sur une période allant de 9MAP jusqu'à 21 MAP comme il en est le cas chez les producteurs, afin de déterminer la période optimale de récolte de chacune d'elle. La meilleure période de récolte correspond au stade végétatif où les racines tubéreuses ont le poids frais et le pourcentage de matière sèche les plus élevés (Osiru et hahn, 1995 cité par B.C. Ebah-Djedji et al. 2012).

Disanka ont été évaluées avec comme témoins les 5 variétés locales réputées comme meilleures dont l'une supposée précoce (Tshilobo), une semi précoce (Lueni), et trois tardives (Kamana Mabanza, Kalenda et Kabuitshi). Pour le prélèvement du poids, un peson de la marque Salter de $100 \mathrm{~kg}$ était utilisé. Un peson électronique portable, un grand seau en plastique et un seau perforé étaient aussi utilisés pour le pesage des échantillons de manioc afin de déterminer les poids spécifiques qui ont permis de calculer la matière sèche ainsi que l'amidon.

Méthodes : Le dispositif expérimental utilisé était celui des blocs complètement randomisés avec trois répétitions où chaque clone avait occupé une parcelle de 9 lignes de 10 plants chacune installés aux écartements de $1 \mathrm{~m} \times 1 \mathrm{~m}$, soit une densité de 10.000 pieds/ha. Les boutures plantées étaient bien aoûtées et mesuraient 20 à $25 \mathrm{~cm}$ de longueur. Les allées entre les répétitions étaient de $2 \mathrm{~m}$. La superficie totale occupée par cet essai était de $7200 \mathrm{~m}^{2}$ dont 1440 d'allées. La parcelle utile comprenait 56 pieds et à chaque récolte 9 plants devaient être considérés en observation. Le champ était maintenu propre par des sarclages manuels jusqu'à la dernière récolte, à 21 MAP. 
Collecte des données; La récolte était effectuée trimestriellement à $9,12,15,18$ et 21 MAP. A chaque récolte, les données suivantes ont été enregistrées: Le Nombre de Plants Récoltés (NPR), le Nombre des Tubercules Frais Commercialisables (NTFC), le Nombre des Tubercules Frais Non Commercialisables (NTFNC), Le Nombre des Tubercules Pourries (NTP), le Poids des Tubercules Frais Commercialisables (PTFC), le Poids des Tubercules Frais Non Commercialisables (PTFNC), poids de la biomasse $(P B)$, le Taux de Matière Sèche(MS) et le Taux d'Amidon(AM).

Le rendement en tubercules (RTF) était estimé suivant la formule :

RTF $=$ poids tubercules $\left(\mathrm{Kg} / \mathrm{m}^{2}\right) \quad \times 10000 / 1000 \mathrm{~kg}$ (Kamau et al., 2010). L'estimation de la teneur en matière sèche et en amidon des variétés en évaluation était faite suivant la méthode de gravité spécifique. Cette méthode consiste à prélever et peser

\section{RÉSULTATS}

Rendement en tubercules frais et Rendement sec : L'évolution de rendement en tubercules frais des différentes variétés est présentée dans le tableau1, simultanément dans l'eau $(\mathrm{Pe})$ et à l'air libre $(\mathrm{Pa})$, pour chaque clone, un échantillon de racines tubéreuses fraiches dont le poids varie entre 300 et $500 \mathrm{~g}$. La gravité spécifique calculée comme suit, $X=\mathrm{Pa} /(\mathrm{Pa}-$ $\mathrm{Pe})$, est introduite dans les équations respectives pour donner les teneurs en matière sèche et en amidon (Fukuda W. M. G et al., 1998) :

Matière sèche $(\%)=158.3^{*} X-142$

Amidon $(\%)=112.1^{*} X-106.4$

Le rendement sec est calculé en multipliant le rendement frais des racines tubéreuses de chaque variété à son pourcentage de Matière Sèche (M.L. Rutikanga et al, 2004, Kamau et al. 2010).

Analyse statistique: L'analyse statistique des données collectées a été effectuée par le biais du test d'analyse de variance (ANOVA) à l'aide du logiciel Statistix 8.0. La mise en évidence des différences significatives entre les traitements a été réalisée au moyen du test LSD au seuil de $5 \%$ de probabilité.

tandis que celle de rendement sec est reprise dans le tableau 2.

Tableau1 : Évolution de rendement en tubercules frais (t/ha) des variétés

\begin{tabular}{l|l|l|l|l|l}
\hline Clone & $\mathbf{9 ~ M A P}$ & $\mathbf{1 2}$ MAP & $\mathbf{1 5}$ MAP & $\mathbf{1 8 ~ M A P}$ & $\mathbf{2 1 ~ M A P}$ \\
\hline Nsansi & $57,7 \mathrm{a}$ & $51,3 \mathrm{a}$ & $66,5 \mathrm{a}$ & $57,7 \mathrm{a}$ & $75,4 \mathrm{a}$ \\
Obama & $31,7 \mathrm{~b}$ & $34,2 \mathrm{abc}$ & $45,6 \mathrm{abc}$ & $33,2 \mathrm{abcd}$ & $51,9 \mathrm{abc}$ \\
Kamana Mabanza & $29,5 \mathrm{~b}$ & $22,8 \mathrm{bc}$ & $54,5 \mathrm{ab}$ & $37,2 \mathrm{abcd}$ & $51,9 \mathrm{abc}$ \\
Kalenda & $28,3 \mathrm{~b}$ & $25,4 \mathrm{bc}$ & $31,4 \mathrm{bc}$ & $26,2 \mathrm{bcd}$ & $35,6 \mathrm{c}$ \\
Zizila & $27,9 \mathrm{~b}$ & $15,6 \mathrm{c}$ & $27,1 \mathrm{bc}$ & $27,3 \mathrm{~cd}$ & $18,6 \mathrm{c}$ \\
Mbankana & $27,8 \mathrm{~b}$ & $29,9 \mathrm{bc}$ & $43,1 \mathrm{abc}$ & $39,8 \mathrm{abcd}$ & $41,9 \mathrm{~b}$ \\
Kabuitshi & $27,5 \mathrm{~b}$ & $27,9 \mathrm{ab}$ & $54,7 \mathrm{ab}$ & $\mathbf{5 9 , 7} \mathrm{a}$ & $38,8 \mathrm{bc}$ \\
Tshilobo & $27,4 \mathrm{~b}$ & $34,1 \mathrm{abc}$ & $44,3 \mathrm{abc}$ & $44,0 \mathrm{abc}$ & $41,9 \mathrm{abc}$ \\
Butamu & $22,7 \mathrm{bc}$ & $23,7 \mathrm{bc}$ & $55 \mathrm{ab}$ & $\mathbf{5 1 , 0} \mathrm{ab}$ & $71, \mathrm{ab}$ \\
Lueni & $22,0 \mathrm{bc}$ & $22,7 \mathrm{bc}$ & $30,9 \mathrm{bc}$ & $19,9 \mathrm{~cd}$ & $19,7 \mathrm{c}$ \\
Disanka & $21,2 \mathrm{bc}$ & $38,6 \mathrm{ab}$ & $45,3 \mathrm{abc}$ & $37,2 \mathrm{abcd}$ & $33,6 \mathrm{c}$ \\
94/0330 & $20,3 \mathrm{bc}$ & $24,9 \mathrm{bc}$ & $22,2 \mathrm{c}$ & $19,9 \mathrm{~cd}$ & $25,6 \mathrm{c}$ \\
Ngandajika & $19,8 \mathrm{bc}$ & $15,8 \mathrm{c}$ & $28,8 \mathrm{bc}$ & $22,0 \mathrm{~d}$ & $32,3 \mathrm{c}$ \\
Mvuazi & $\mathbf{9 , 8 \mathrm { c }}$ & $18,5 \mathrm{c}$ & $\mathbf{2 8 , 3} \mathrm{bc}$ & $14,8 \mathrm{~d}$ & $33,3 \mathrm{c}$ \\
\hline Grand Mean & $\mathbf{2 6 , 7}$ & $\mathbf{2 7 , 4}$ & $\mathbf{4 1 , 3}$ & $\mathbf{3 4 , 6}$ & $\mathbf{4 0 , 8}$ \\
CV(\%) & $\mathbf{2 7 , 4 3}$ & $\mathbf{4 3 , 8}$ & $\mathbf{4 5 , 6 3}$ & $\mathbf{4 6 , 4 5}$ & $\mathbf{4 9 , 0 6}$ \\
LSD(0,05) & $\mathbf{1 2 , 2 8}$ & $\mathbf{5 , 1 3 3}$ & $\mathbf{3 1 , 6 0}$ & $\mathbf{2 6 , 9 6}$ & $\mathbf{3 3 , 5 9}$ \\
\hline
\end{tabular}

Les moyennes suivies d'une même lettre dans la même colonne ne diffèrent pas significativement au seuil de $5 \%$ de probabilité selon le test $L S D(p>0,05)$ 
Tableau 2 : Évolution du rendement sec (t/ha) des variétés

\begin{tabular}{|c|c|c|c|c|c|}
\hline Clone & 9 MAP & 12 MAP & 15 MAP & 18 MAP & 21 MAP \\
\hline Nsansi & $12,9 \mathrm{a}$ & $17,6 a$ & $19,1 \mathrm{a}$ & $12,2 a b$ & $15,3 \mathrm{a}$ \\
\hline Kamana Mabanza & $7,6 \mathrm{~b}$ & $7,7 \mathrm{~b}$ & $17,0 a b c$ & $10,0 \mathrm{abcd}$ & $11,8 a b$ \\
\hline Mbankana & $7,4 \mathrm{~b}$ & $9,1 \mathrm{~b}$ & $14,4 \mathrm{abc}$ & 9,7 abcd & $9,5 a b c$ \\
\hline Kalenda & $7,3 b$ & $8,6 b$ & $10,0 \mathrm{abc}$ & $6,7 \mathrm{bcd}$ & $6,7 \mathrm{bc}$ \\
\hline Obama & $6,9 b c$ & $11,5 a b$ & $17,0 \mathrm{abc}$ & $9,2 \mathrm{abcd}$ & $11,0 a b c$ \\
\hline Butamu & $6,8 \mathrm{bc}$ & $7,6 \mathrm{~b}$ & $19,0 a b$ & $12,0 a b$ & $15,3 \mathrm{a}$ \\
\hline Tshilobo & $6,8 b c$ & $10,6 a b$ & $15,6 a b c$ & 9,5 abcd & $7,8 \mathrm{bc}$ \\
\hline Ngandajika & $6,7 \mathrm{bc}$ & $5,3 \mathrm{~b}$ & $9,8 \mathrm{abc}$ & 5,6 bcd & $6,2 \mathrm{bc}$ \\
\hline Disanka & $6,3 \mathrm{bc}$ & $12,2 a b$ & $14,0 a b c$ & $6,6 \mathrm{bcd}$ & $6,7 \mathrm{bc}$ \\
\hline Zizila & $6,0 \mathrm{bc}$ & $5,2 \mathrm{~b}$ & 8,7 bc & $5,3 \mathrm{~cd}$ & $3,7 \mathrm{c}$ \\
\hline Kabuitshi & $6,0 \mathrm{bc}$ & $8,8 \mathrm{~b}$ & $20,1 \mathrm{a}$ & $15,5 \mathrm{a}$ & $8 a b c$ \\
\hline Vuvu & $5,1 \mathrm{bc}$ & $7,0 \mathrm{~b}$ & $8,2 \mathrm{c}$ & $4,7 d$ & $5,7 \mathrm{bc}$ \\
\hline Lueni & 4,8 bc & $7,5 b$ & $9,4 a b c$ & $4,1 d$ & $3,7 \mathrm{c}$ \\
\hline Mvuazi & $2,9 \mathrm{c}$ & $6,2 \mathrm{~b}$ & $9,4 a b c$ & $3,6 \mathrm{~d}$ & $6,4 \mathrm{bc}$ \\
\hline Moyenne & 6,7 & 8,9 & 13,7 & 8,2 & 8,4 \\
\hline CV(\%) & 38,78 & 47,49 & 46,98 & 50,36 & 51,82 \\
\hline $\operatorname{LSD}(0,05)$ & 4,341 & 7,12 & 10,84 & 6,94 & 7,34 \\
\hline
\end{tabular}

Les moyennes suivies d'une même lettre dans la même colonne ne diffèrent pas significativement au seuil de $5 \%$ de probabilité selon le test $L S D(p>0,05)$

II ressort de la lecture des résultats sur le rendement en tubercules frais et le rendement sec consignés respectivement dans les tableaux 1 et 2 , que les clones se sont comporté des façons variées. Entre 9 MAP et 12 MAP, l'accroissement du rendement en tubercules frais et du rendement sec n'est pas très significatif et chez certains clones une diminution est observée.

Teneur en Matière sèche et en Amidon : L'évolution du taux de la matière sèche des variétés et celle de l'amidon sont présentées dans les tableaux 3 et 4 suivants :

Tableau3 : Évolution de taux de la Matière sèche (\%)

\begin{tabular}{l|l|l|l|l|l}
\hline Clone & $\mathbf{9 ~ M A P}$ & $\mathbf{1 2}$ MAP & 15 MAP & 18 MAP & 21 MAP \\
\hline Kabuitshi & $21,9 \mathrm{~b}$ & $31,2 \mathrm{ab}$ & $34,3 \mathrm{ab}$ & $25,7 \mathrm{ab}$ & $20,4 \mathrm{a}$ \\
Ngandajika & $34,0 \mathrm{a}$ & $33,6 \mathrm{a}$ & $35,1 \mathrm{ab}$ & $25,1 \mathrm{ab}$ & $19,2 \mathrm{a}$ \\
Butamu & $29,1 \mathrm{ab}$ & $33,2 \mathrm{ab}$ & $32,7 \mathrm{ab}$ & $24,5 \mathrm{abc}$ & $21,4 \mathrm{a}$ \\
94/330 & $24,8 \mathrm{ab}$ & $28,5 \mathrm{~b}$ & $36,0 \mathrm{ab}$ & $25,3 \mathrm{ab}$ & $22,4 \mathrm{a}$ \\
Nsansi & $22,4 \mathrm{ab}$ & $34,2 \mathrm{a}$ & $30,2 \mathrm{~b}$ & $20,8 \mathrm{~cd}$ & $19,0 \mathrm{a}$ \\
Kalenda & $25,9 \mathrm{ab}$ & $34,4 \mathrm{a}$ & $31,6 \mathrm{ab}$ & $26,3 \mathrm{a}$ & $19,7 \mathrm{a}$ \\
Disanka & $27,9 \mathrm{ab}$ & $31,6 \mathrm{ab}$ & $30,9 \mathrm{ab}$ & $17,6 \mathrm{~d}$ & $19,7 \mathrm{a}$ \\
Mbankana & $26,2 \mathrm{ab}$ & $31,5 \mathrm{ab}$ & $32,6 \mathrm{ab}$ & $24,3 \mathrm{abc}$ & $22,9 \mathrm{a}$ \\
Kamana M. & $25,4 \mathrm{ab}$ & $33,9 \mathrm{a}$ & $31,8 \mathrm{ab}$ & $27,2 \mathrm{a}$ & $23,1 \mathrm{a}$ \\
Mvuazi & $24,7 \mathrm{ab}$ & $34,4 \mathrm{a}$ & $33,3 \mathrm{ab}$ & $24,0 \mathrm{abc}$ & $20,8 \mathrm{a}$ \\
Tshilobo & $23,7 \mathrm{~b}$ & $31,7 \mathrm{ab}$ & $35,1 \mathrm{ab}$ & $21,9 \mathrm{bc}$ & $19,0 \mathrm{a}$ \\
Zizila & $21,9 \mathrm{~b}$ & $33,2 \mathrm{ab}$ & $32,1 \mathrm{ab}$ & $20,8 \mathrm{~cd}$ & $20,4 \mathrm{a}$ \\
Obama & $21,8 \mathrm{~b}$ & $33,7 \mathrm{ab}$ & $37,0 \mathrm{a}$ & $27,2 \mathrm{a}$ & $21,7 \mathrm{a}$ \\
Lueni & $21,5 \mathrm{~b}$ & $33,7 \mathrm{a}$ & $32,4 \mathrm{ab}$ & $20,5 \mathrm{~cd}$ & $18,9 \mathrm{a}$ \\
\hline Grand Means & 25,1 & 32,7 & 33,2 & 23,7 & 20,6 \\
CV(\%) & 24,8 & 9,35 & 11,18 & 10,08 & 12,2 \\
LSD(0,05) & 10,43 & 5,133 & 6,218 & 4,002 & \\
\hline
\end{tabular}

Les moyennes suivies d'une même lettre dans la même colonne ne diffèrent pas significativement au seuil de $5 \%$ de probabilité selon le test $L S D(p>0,05)$ 

manioc (Manihot esculenta Crantz) tant locales qu'améliorées cultivées à Ngandajika en RD Congo

Tableau4 : Evolution du taux d'amidon (\%)

\begin{tabular}{l|l|l|l|l|l}
\hline Clone & $\mathbf{9 ~ M A P}$ & $\mathbf{1 2}$ MAP & $\mathbf{1 5}$ MAP & $\mathbf{1 8 ~ M A P}$ & 21 MAP \\
\hline Kabuitshi & $9,7 \mathrm{~b}$ & $16,2 \mathrm{ab}$ & $18,6 \mathrm{ab}$ & $12,3 \mathrm{ab}$ & $8,6 \mathrm{ab}$ \\
Ngandajika & $18,2 \mathrm{a}$ & $18,0 \mathrm{a}$ & $19,2 \mathrm{ab}$ & $11,9 \mathrm{ab}$ & $7,7 \mathrm{ab}$ \\
Butamu & $14,7 \mathrm{ab}$ & $17,0 \mathrm{ab}$ & $17,4 \mathrm{ab}$ & $10,8 \mathrm{abc}$ & $9,3 \mathrm{ab}$ \\
$94 / 330$ & $11,7 \mathrm{ab}$ & $14,3 \mathrm{~b}$ & $19,2 \mathrm{ab}$ & $12,1 \mathrm{ab}$ & $10,0 \mathrm{ab}$ \\
Nsansi & $10,0 \mathrm{ab}$ & $18,4 \mathrm{a}$ & $15,7 \mathrm{~b}$ & $8,9 \mathrm{~cd}$ & $8,0 \mathrm{ab}$ \\
Kalenda & $12,9 \mathrm{ab}$ & $18,5 \mathrm{a}$ & $16,7 \mathrm{ab}$ & $12,8 \mathrm{a}$ & $7,6 \mathrm{ab}$ \\
Disanka & $13,9 \mathrm{ab}$ & $16,6 \mathrm{ab}$ & $16,2 \mathrm{ab}$ & $6,6 \mathrm{~d}$ & $8,1 \mathrm{ab}$ \\
Mbankana & $12,7 \mathrm{ab}$ & $16,5 \mathrm{ab}$ & $17,4 \mathrm{ab}$ & $11,4 \mathrm{abc}$ & $10,4 \mathrm{ab}$ \\
Kamana M. & $12,1 \mathrm{ab}$ & $18,1 \mathrm{a}$ & $16,8 \mathrm{ab}$ & $13,4 \mathrm{a}$ & $10,5 \mathrm{a}$ \\
Mvuazi & $11,6 \mathrm{ab}$ & $18,6 \mathrm{a}$ & $17,9 \mathrm{ab}$ & $11,1 \mathrm{abc}$ & $8,9 \mathrm{ab}$ \\
Tshilobo & $10,7 \mathrm{~b}$ & $16,6 \mathrm{ab}$ & $19,1 \mathrm{ab}$ & $9,7 \mathrm{bc}$ & $8,6 \mathrm{ab}$ \\
Zizila & $9,7 \mathrm{~b}$ & $17,7 \mathrm{ab}$ & $17 \mathrm{ab}$ & $9,6 \mathrm{bc}$ & $8,6 \mathrm{ab}$ \\
Obama & $9,6 \mathrm{~b}$ & $18,0 \mathrm{a}$ & $20,5 \mathrm{a}$ & $13,4 \mathrm{a}$ & $9,5 \mathrm{ab}$ \\
Lueni & $9,4 \mathrm{~b}$ & $18,1 \mathrm{a}$ & $17,2 \mathrm{ab}$ & $8,7 \mathrm{~cd}$ & $7,5 \mathrm{~b}$ \\
\hline Grand Means & 11,9 & 17,3 & 17,8 & 10,9 & 8,7 \\
CV(\%) & 36,94 & 12,55 & 14,7 & 15,4 & 20,26 \\
LSD(0,05) & 7,372 & 3,644 & 4,409 & 2,847 & 2,973 \\
\hline
\end{tabular}

Les moyennes suivies d'une même lettre dans la même colonne ne diffèrent pas significativement au seuil de $5 \%$ de probabilité selon le test $L S D(p>0,05)$

Les résultats sur les taux de la Matière Sèche et d'Amidon consignés dans le tableau 3 et 4 indiquent que les taux de MS et d'AM les plus élevés sont obtenus à partir de 12 MAP, ils restent presque stable jusqu' à 15 MAP et décroissent à partir de 18 MAP. A 9 MAP, le taux le plus élevé était obtenu par la variété Ngandajika (34\%) tandis que la variété locale Lueni a eu le taux le plus bas $(21,5 \%)$. A 18 MAP, les variétés

\section{DISCUSSION}

II ressort des résultats obtenus dans le tableau 1 et 2, sur le rendement en tubercules frais et le rendement sec que les clones se sont comporté des façons variées. Entre $9 \mathrm{MAP}$ et $12 \mathrm{MAP}$, l'accroissement du rendement en tubercules frais et du rendement sec n'est pas très significatif et chez certains clones une diminution est observée. Des résultats pareils étaient obtenus par Tewodros Mulualem et Jimma en 2012. Entre 12 MAP et 15 MAP, presque toutes les variétés ont manifesté un accroissement de rendement frais et de poids secs tandis qu'entre 15 MAP et 18 MAP, la tendance à la baisse est constatée chez presque tous les clones. Les résultats similaires ont été obtenus dans le passé (Ngendahayo et Dixon.2 001, Bakayoko et al. 2012, Michael A. Edet et al. 2015). Selon ces travaux, la période optimum de récolte, dépend des variétés et de facteurs écologiques. La grandeur des racines augmente avec l'âge de plants jusqu'à 15 MAP avec une diminution progressive de la production des améliorés Obama, Ngandajika, Butamu, Vuvu, Mbankana et Mvuazi ne sont pas significativement différentes des variétés Kamana Mabanza et Kalenda qui sont réputées tardives et considérées comme ayant une meilleure conservation dans le sol. A 21 MAP, il n'y a eu aucune différence significative entre les variétés.

racines peu avant cette période (Ngendahayo and Dixon, 2001, cités par Bassey et Harry). Michael A. Edet et al notent que, récolter le manioc au-delà de 15 MAP ne contribue pas à une augmentation significative du rendement final parce que la plante va réutiliser les réserves des racines pour l'émission et la croissance des repousses avec comme conséquence la réduction du rendement et de la qualité des tubercules. En 2012, Tewodors et Jimma avaient trouvé que les rendements élevés en tubercules frais étaient obtenus à $24 \mathrm{MAP}$, mais le problème de la qualité de la farine dû à l'accumulation de la cellulose se posait. II sied de noter que la récolte à 18 MAP était effectuée au mois de juillet en pleine saison sèche et celle à 21 MAP au Mois d'octobre, 2 mois après le retour des pluies. Le rétablissement du fonctionnement physiologique normal de la plante avec le retour des pluies expliquerait aussi l'augmentation $\mathrm{du}$ rendement en tubercules frais enregistrée chez certaines variétés. Githunguri et al. 

manioc (Manihot esculenta Crantz) tant locales qu'améliorées cultivées à Ngandajika en RD Congo

2004 cité par Bassey et Harry en 2013 ont reporté que dans les régions où il pleut beaucoup le taux de grossissement des tubercules est plus élevé que celui des régions où il pleut moins. Considérant la performance individuelle de chaque clone, on note que la variété améliorée Nsansi, a obtenu les rendements en tubercules frais et les rendements secs les plus élevés à presque tous les âges, excepté à 18 MAP où la variété locale Kabuitshi $(59,7$ t/ha et 15,5 t/ha) était en tête. A 9 MAP, La variété Nsansi dépasse

\section{CONCLUSION ET RECOMMENDATIONS}

Considérant la définition de l'âge propice de récolte tel que défini par Osiru et Hahn, 1995, qui stipule que la meilleure période de récolte du manioc correspond au stade végétatif où le rendement en tubercules frais et le taux de la matière sèche sont les plus élevés, la période optimum de récolte pour toutes ces variétés (locales et améliorées) se situerait entre 12 MAP et 15 MAP. La précocité ou la tardivité des variétés locales, selon la considération paysanne n'ont pas été confirmées par ce travail. La variété Nsansi a manifesté une maturité élastique pouvant aller de précoce à tardive. Eu égard à l'évolution de son taux de matière sèche et du poids frais de ses racines tubéreuses, la variété Ngandajika s'est aussi montrée précoce. Vu leurs résultats à $18 \mathrm{MAP}$, les variétés améliorées, Obama, Ngandajika, Butamu, Vuvu,

\section{RÉFÉRENCES BIBLIOGRAPHIQUES}

Ashoka PV, Nair SV, Kurian. TM (1984). Influence of stages of harvest on the yield and quality of cassava (Manihot esculenta crantz). Mandraos Agric.j. 71: 447-449

Bakayoko S., Kouadio K.K.H., Soro D., Tschannen A., Nindjin C., Dao D, Girardin O. Rendements en tubercules frais et teneurs en matière sèche de soixante-dix nouvelles variétés de manioc (Manihot esculenta Crantz) cultivées dans le centre de la Côte d'Ivoire.

Benesi, I. R. M., M. T. et N.M. Mahungu, 2004. Genotype $x$ Environment interaction effects on native cassava starch quality and potential for starch use in the commercial stector. African Journal of Crop Science 12:205-216.

B.C. Ebah-Djedji, K.M. Dje, B.N'Zue, G.P.Zohouri et N.G.Amani, 2012. Effect of harvest period on Starch Yield and Dry Matter Content from the Tuberous Roots of Improved Cassava (Manihot esculenta Crantz) varieties. significativement toutes les variétés avec ses 57,7 t/ha de rendement en tubercules frais et 12,9 de rendement sec. La variété Mvuazi (9,8 t/ha et 2,9 t/ha) a obtenu les rendements frais et sec les plus bas. A 12 MAP, la variété Nsansi dépasse encore de façon significative tous les clones à l'exception des variétés Obama, Tshilobo et Disanka. Les variétés Kalenda, Zizila, Lueni, Vuvu, Ngandajika et Mvuazi lui sont significativement inférieur jusqu'à la dernière récolte à 21 MAP.

Mbankana et Mvuazi sont similaires aux variétés locales Kamana Mabanza et Kalenda qui sont réputées tardives et considérées comme ayant une meilleure conservation de tubercules dans le sol et peuvent être conseillées aux partisans d'une récolte échelonnée. Etant donné que la période optimale de la récolte se situe entre 12 et $15 \mathrm{MAP}$, nous recommandons que la récolte de toutes ces variétés soit faite au plus tard à 15 MAP. Pour les agriculteurs de l'hinterland de la cité de Ngandajika qui préfèrent les variétés récoltables à 12 MAP, nous recommandons toutes ces variétés mais particulièrement les variétés Nsansi, Ngandajika, Disanka et Vuvu. Pour les agriculteurs des autres villages qui ont suffisamment de terres, nous recommandons d'utiliser toutes les variétés sans dépasser la période de 15 MAP.

Emmanuel E. Bassey and Gamaliel I. Harry, 2013. Screening cassava (Manihot esculenta Crantz) genotypes for tuber bulking, early maturity and optimum harvesting time in Uyo, South Eastern Nigeria. Peak journal of Agricultural Sciences vol. 1(5), 83-88.

Fargette D., Fauquet C. et Thouvenel J-C., 1988. Yield losses induced by African cassava mosaic virus in relation to the mode and the date of infection. Tropical pest management, 34 (1) 89-91.

Hahn SK. Terry ER, Leushner K. Akobundu IO. Okali C. Lal R. 1979. Cassava improvement in Africa. Field Crops Res.23 : 193-226.

IITA-DRC, Projet Recherche Développement D'appui à I'Industrie du Manioc en R.D.C., programme d'activités 2012-2013.

IRAZ (Institut de Recherche Agronomique et Zootechnique de la CEPGL), 1989. Étude pédologique de huit sites repères pour les essais au sein de la CEPGL (Moso, Mashitsi, 
Rubona, Karama, Yangambi, Mulungu, Gandajika, M'vuazi).

J. Kamau, R. Melis, Mark Laing, J. Derera, P. Shanahan et Eliud C.K.Ngugi. 2011. Farmers 'participatory selection for early Bulking Cassava genotypes in semi-arid Eastern Kenya. Journal of Plant Breeding and crop sciences vol. 3(3), PP. 44-52.

Michael A. Edet, H. Tijani-Eniola, S. T. O. Lagoke, Gbassay Tarawali, 2015. Relationship of Cassava Growth Parameters with Yield, Yield related components and Harvest Time in Ibadan, Southwestern Nigeria. Journal of Natural Sciences Research, ISSN 2224-3186, vol5, № 9.

M.L. Rutikanga, D.S.O.Osiru, J.K. Makelele et C.Bayituliki, 2004. Effects of location and age at harvest on the yield of cassava genotypes (Manihot esculenta Crantz) in Ruanda. Proceedings: 9th ISTRC-AB Symposium, Kenya, page 507.

M. Muengula M, 2010. l'évaluation en milieu paysan du comportement de six variétés de manioc d'introduction récente et résistantes à la mosaïque africaine. UNIKIN, Thèse DEA.

Ntawurunga P. Ojulong H., Dixon AGO (1998). Genetic variability among cassava genotypes and its growth performance over time in Root crops and poverty alleviation. Proccedings of the $6^{\text {th }}$ Symposium of ISTRC-African Branch IITA. Ibadan, Nigeria, PP 242-248.

Tata Hangy et Phemba Pezo, 2002. Guide pratique pour les agents de terrain, GLCl, CRS, IITA, deus entreprises et UCAEC.

Tewodros Mulualem et Biruk Ayenew, 2012. Cassava (Manihot esculenta Crantz) Varieties and Harvesting stages Influenced Yield and Yield related Components. Journal of Natural Sciences Research, ISSN 2224-3186, Vol 2, No10.

W. M. G. Fukuda, C. L. Guevara, M. E. Ferguson and R. Kawuki. 1998. Descriptors for cassava Morphological Characterisation (EMBRAPACNPMF, Documentos, 78). 